\title{
Robust de novo design of protein binding proteins from target structural information alone
}

Longxing Cao ${ }^{1,2, \# \text {, Brian Coventry }}{ }^{1,2,3, \#}$, Inna Goreshnik ${ }^{1,2}$, Buwei Huang ${ }^{1,2,4}$, Joon Sung Park $^{5}$, Kevin M. Jude ${ }^{6,7,8}$, Iva Marković9,10, Rameshwar U. Kadam ${ }^{11}$, Koen H.G. Verschueren ${ }^{9,10}$, Kenneth Verstraete ${ }^{9,10}$, Scott Thomas Russell Walsh ${ }^{12,13}$, Nathaniel Bennett ${ }^{1,2,3}$, Ashish Phal ${ }^{1,4,18}$, Aerin Yang ${ }^{6,7,8}$, Lisa Kozodoy ${ }^{1,2}$, Michelle DeWitt ${ }^{1,2}$, Lora Picton $^{6,7,8}$, Lauren Miller ${ }^{1,2}$, Eva-Maria Strauch ${ }^{14}$, Samer Halabiya ${ }^{15}$, Bradley Hammerson ${ }^{16}$, Wei Yang ${ }^{1,2}$, Steffen Benard ${ }^{11}$, Lance Stewart ${ }^{1,2}$, lan A. Wilson ${ }^{11,17}$, Hannele Ruohola-Baker ${ }^{1,18}$, Joseph Schlessinger ${ }^{5}$, Sangwon Lee ${ }^{5}$, Savvas N. Savvides $^{9,10}$, K. Christopher Garcia ${ }^{6,7,8}$, David Baker ${ }^{1,2,19^{*}}$

1. Department of Biochemistry, University of Washington, Seattle, WA 98195, USA.

2. Institute for Protein Design, University of Washington, Seattle, WA 98195, USA.

3. Molecular Engineering Graduate Program, University of Washington, Seattle, WA 98195, USA.

4. Department of Bioengineering, University of Washington, Seattle, WA, 98195, USA.

5. Department of Pharmacology, Yale University School of Medicine, New Haven, CT 06520, USA.

6. Howard Hughes Medical Institute, Stanford University School of Medicine, Stanford, CA 94305, USA.

7. Department of Structural Biology, Stanford University School of Medicine, Stanford, CA 94305, USA.

8. Department of Molecular and Cellular Physiology, Stanford University School of Medicine, Stanford, CA 94305, USA.

9. VIB-UGent Center for Inflammation Research, 9052 Ghent, Belgium

10. Unit for Structural Biology, Department of Biochemistry and Microbiology, Ghent University, 9052 Ghent, Belgium.

11. Department of Integrative Structural and Computational Biology, The Scripps Research Institute, La Jolla, CA 92037, USA.

12. National Cancer Institute, National Institutes of Health, Chemical Biology Laboratory, 1050 Boyles Street, Building 376, Frederick, MD 21702

13. Present address: J.A.M.E.S. Farm, 13615 Highland Road, Clarksville, MD 21029, USA

14. Dept. of Pharmaceutical and Biomedical Sciences, University of Georgia, Athens, GA 30602, USA

15. Department of Electrical and Computer Engineering, University of Washington, Seattle, WA 98195, United States.

16. Seattle Structural Genomics Center for Infectious Disease, Seattle, WA 98109, USA. 
17. The Skaggs Institute for Chemical Biology, The Scripps Research Institute, La Jolla, CA 92037, USA.

18. Institute for Stem Cell and Regenerative Medicine, University of Washington, Seattle, WA 98109, USA

19. Howard Hughes Medical Institute, University of Washington, Seattle, WA 98195, USA \#These authors contributed equally

\section{Abstract}

The design of proteins that bind to a specific site on the surface of a target protein using no information other than the three-dimensional structure of the target remains an outstanding challenge. We describe a general solution to this problem which starts with a broad exploration of the very large space of possible binding modes

55 and interactions, and then intensifies the search in the most promising regions. We demonstrate its very broad applicability by de novo design of binding proteins to 12

57 diverse protein targets with very different shapes and surface properties. Biophysical

58 characterization shows that the binders, which are all smaller than 65 amino acids, are

59 hyperstable and bind their targets with nanomolar to picomolar affinities. We succeeded

60 in solving crystal structures of four of the binder-target complexes, and all four are very

61 close to the corresponding computational design models. Experimental data on nearly

62 half a million computational designs and hundreds of thousands of point mutants

63 provide detailed feedback on the strengths and limitations of the method and of our

64 current understanding of protein-protein interactions, and should guide improvement of

65 both. Our approach now enables targeted design of binders to sites of interest on a 66 wide variety of proteins for therapeutic and diagnostic applications. 


\section{Introduction}

68 Protein interactions play critical roles in biology, and general approaches to

69 disrupt or modulate these with designed proteins would have huge impact. While

70 empirical laboratory selection approaches starting from very large antibody, DARPIN or

71 other protein scaffold libraries can generate binders to protein targets, it is difficult at the

72 outset to target a specific region on a target protein surface, and to sample the full

73 space of possible binding modes. Computational methods can target specific target

74 surface locations and provide a more principled and potentially much faster approach to

75 binder generation than random library selection methods, as well as insight into the

76 fundamental properties of protein interfaces (which must be understood for design to be

77 successful). Most current methods for computationally designing proteins to bind to a

78 target surface utilize information derived from native complex structures on specific

79 sidechain interactions or protein backbone placements optimal for binding ${ }^{1-3}$.

80 Computational docking of antibody scaffolds with varied loop geometries has yielded

81 binders, but the designed binding modes have rarely been validated with high-resolution

82 structures ${ }^{4}$. Binders have been generated starting from several computationally

83 identified hot-spot residues, which were then used to guide the positioning of naturally

84 occurring protein scaffolds ${ }^{5}$. However, for many target proteins, there are no obvious

85 pockets or clefts on the protein surface into which a small number of privileged

86 sidechains can be placed, and guidance by only a small number of hotspot residues

87 limits the approach to a small fraction of possible interaction modes. 


\section{Design Method}

We sought to develop a general approach to design of high affinity binders to arbitrary protein targets that addresses two major challenges. First, in the general case,

91 there are no clear sidechain interactions or secondary structure packing arrangements

92 that can mediate strong interactions with the target; instead there are a very large

93 number of individually very weak possible interactions. Second, the number of ways of

94 choosing from these numerous weak interactions to incorporate into a single binding

95 protein is combinatorially large, and any given protein backbone is unlikely to be able to

96 simultaneously present sidechains that can encompass any preselected subset of these

97 interactions. To motivate our approach, consider the simple analogy of a very difficult

98 climbing wall with only a few good footholds or handholds distant from each other.

99 Previous "hotspot" based approaches correspond to focusing on routes involving these

100 footholds/handholds, but this greatly limits the possibilities and there may be no way to

101 connect them into a successful route. An alternative is to first, identify all possible

102 handholds and footholds, no matter how poor, second, have thousands of climbers

103 select subsets of these, and try to climb the wall, third, identify those routes that were

104 most promising, and fourth, have a second group of climbers explore them in detail.

105 Following this analogy, we devised a multi-step approach to overcome the above two

106 challenges by 1) enumerating a large and comprehensive set of disembodied sidechain

107 interactions with the target surface, 2) identifying from large in silico libraries of protein

108 backbones those that can host many of these sidechains without clashing with the

109 target, 3) identifying recurrent backbone motifs in these structures, and 4) generating

110 and placing against the target a second round of scaffolds containing these interacting 
111 motifs (Fig. 1a). Steps 1 and 2 search the space very widely, while steps 3 and 4

112 intensify search in the most promising regions. We describe and motivate each step in

113 the following paragraphs.

We began by docking disembodied amino acids against the target protein, and

116 storing the backbone coordinates and target binding energies of the typically billions of

117 amino acids making favorable hydrogen bonding or non-polar interactions in a 6-

118 dimensional spatial hash table for rapid lookup (Fig. 1a; see methods). This "rotamer

119 interaction field" (RIF) enables rapid approximation of the target interaction energy

120 achievable by a protein scaffold docked against a target based on its backbone

121 coordinates alone (with no need for time consuming sidechain sampling)--for each dock,

122 the target interaction energies of each of the matching amino acids in the hash table are

123 summed. A related approach was used for small molecule binder design'; since protein

124 targets are so much bigger, and non-polar interactions are the primary driving force for

125 protein-protein association, we focused the RIF generation process on non-polar sites in

126 specific surface regions of interest: for example in the case of inhibitor design,

127 interaction sites with biological partners. The RIF approach improves upon previous

128 discrete interaction-sampling approaches ${ }^{5}$ by reducing algorithmic complexity from $\mathrm{O}(\mathrm{N})$

129 or $\mathrm{O}\left(\mathrm{N}^{2}\right)$ to $\mathrm{O}(1)$ with respect to the number of sidechain-target interactions considered,

130 allowing for billions, rather than thousands, of potential interfaces to be considered.

For docking against the rotamer interaction field, it is desirable to have a very

133 large set of protein scaffold options, as the chance that any one scaffold can house 
134 many interactions is small. The structure models of these scaffolds must be quite

135 accurate so that the positioning is correct. Using fragment assembly ${ }^{7}$, piecewise

136 fragment assembly ${ }^{8}$, and helical extension ${ }^{9}$, we designed a large set of miniproteins

137 ranging in length from 50 to 65 amino acids containing larger hydrophobic cores than

138 previous miniprotein scaffold libraries ${ }^{1}$, which makes the protein more stable and more

139 tolerant to introduction of the designed binding surfaces. 84,690 scaffolds spanning 5

140 different topologies with structural metrics predictive of folding were encoded in large

141 oligonucleotide arrays and 34,507 were found to be stable using a high-throughput

142 proteolysis based protein stability assay ${ }^{10}$.

We experimented with several approaches for docking these stable scaffolds

145 against the target structure rotamer interaction field, balancing overall shape

146 complementarity with maximizing specific rotamer interactions. The most robust results

147 were obtained using direct low resolution shape matching ${ }^{11}$ followed by grid based

148 refinement of the rigid body orientation in the RIF (RIFDock). This resulted in better

149 Rosetta binding energies (ddGs) and packing (contact molecular surface, see below)

150 after sequence design than shape matching alone with PatchDock (Fig. 1b red and

151 green), and more extensive non polar interaction with the target than hierarchical

152 search without PatchDock shape matching ${ }^{6}$ (Extended Data Fig. 1). necessarily approximate accounting for interactions between sidechains (see methods),

156 we found that evaluation of the RIF solutions is considerably enhanced by full 
157 combinatorial optimization using the Rosetta forcefield, allowing the target sidechains to

158 repack and the scaffold backbone to relax. Full combinatorial sequence optimization is

159 quite CPU intensive, however, and to enable rapid screening through millions of

160 alternative backbone placements, we developed a rapid pre-screening method using

161 Rosetta to identify promising RIF docks. We found that including only hydrophobic

162 amino acids, using a reduced set of rotamers than in standard Rosetta design

163 calculations, and a more rapidly computable energy function sped design more than 10-

164 fold while retaining a strong correlation with results after full sequence design (next

165 paragraph); this pre-screen (referred to as the "Predictor" below) substantially improved

166 the binding energies and shape complementarity of the final designs as far more RIF

167 solutions could be processed (Extended Data Fig. 2).

We observed that application of standard Rosetta design to the set of filtered

170 docks in some cases resulted in models with buried unsatisfied polar groups and other

171 suboptimal properties. To overcome these limitations, we developed a combinatorial

172 sequence design protocol that maximizes shape and chemical complementarity with the

173 target while avoiding buried polar atoms. Sequence compatibility with the scaffold

174 monomer structure was increased using a structure based sequence profile ${ }^{12}$, the

175 cross-interface interactions were upweighted during the Monte Carlo-based sequence

176 design stage to maximize the contacts between the binder and the target

177 (ProteinProteinInterfaceUpweighter; see Methods), and rotamers containing buried

178 unsatisfiable polar atoms were eliminated prior to packing and buried unsatisfied polar

179 atoms penalized by a pair-wise decomposable pseudo-energy term ${ }^{13}$. This protocol 
180 yielded amino acid sequences more strongly predicted to fold to the designed structure

181 (Extended Data Fig. 3a) and to bind the target (Extended Data Fig. 3b) than standard

182 Rosetta interface design.

In the course of developing the overall binder design pipeline, we noticed upon

185 inspection that even designs with favorable Rosetta binding free energies, large

186 changes in Solvent Accessible Surface Area (SASA) upon binding, and high shape

187 complementarity (SC) often lacked dense packing and interactions involving several

188 secondary structural elements. We developed a quantitative measure of packing quality

189 in closer accord with visual assessment -- the contact molecular surface (see methods)

190 -- which balances interface complementarity and size in a manner that explicitly

191 penalizes poor packing. We used this metric to help select designs at both the rapid

192 Predictor stage and after full sequence optimization (see Methods).

The space sampled by the search over structure and sequence space is

195 enormous: tens of thousands of possible protein backbones $\times$ nearly one billion possible

196 disembodied sidechain interactions per target $\times 10^{16}$ interface sequences per scaffold

197 placement. Sampling of spaces of this size is necessarily incomplete, and many of the

198 designs at this stage contained buried unsatisfied polar atoms (only rotamers that

199 cannot make hydrogen bonds in any context are excluded at the packing stage) and

200 cavities. To generate improved designs, we intensified the search around the best of the

201 designed interfaces. We developed a resampling protocol which extracts all the 202 secondary structural motifs making good contacts with the target protein from the first 
203 "broad search" designs, clusters these motifs based on their backbone coordinates and 204 rigid body placements, and then selects the binding motif in each cluster with the best

205 per-position weighted Rosetta binding energy; around 2,000 motifs were selected for 206 each target. These motifs, which are privileged because they contain a much greater 207 density of favorable side chain interactions with the target than the rest of the designs, 208 were then used to guide another round of docking and design. Scaffolds from the 209 library were superimposed on the privileged motifs, the favorable-interacting motif 210 residues transferred to the scaffold, and the remainder of the scaffold sequence 211 optimized to make further interactions with the target, allowing backbone flexibility to

212 increase shape complementarity with the target (Fig. 1a). Interface metrics for the 213 designs based on the resampling protocol were considerably improved relative to those 214 of the designs from the broad searching stage (Fig. 1b). The designs with the most 215 favorable protein folding and protein interface metrics from both the broad searching 216 and resampling stages were selected for experimental validation.

\section{Experimental testing}

219 targets, which limits assessment of their generality. To robustly test our new binder 220 design pipeline, we selected thirteen native proteins of considerable current interest 221 spanning a wide range of shapes and biological functions. These proteins fall into two 222 classes: first, human cell surface or extracellular proteins involved in signaling, for 223 which binders could have utility as probes of biological mechanism and potentially as

224 therapeutics (Tropomyosin receptor kinase A (TrkA) ${ }^{14}$, Fibroblast growth factor receptor 225 2 (FGFR2) $^{15}$, Epidermal growth factor receptor (EGFR) ${ }^{16}$, Platelet-derived growth factor 
226 receptor (PDGFR) ${ }^{17}$, Insulin receptor $(\text { InsulinR })^{18}$, Insulin-like growth factor 1 receptor

$227(\text { IGF1R })^{19}$, Angiopoietin-1 receptor $(\text { Tie2 })^{20}$, Interleukin-7 receptor alpha (IL-7Ra $)^{21}$, CD3

228 delta chain $(\mathrm{CD} 3 \delta)^{22}$, Transforming growth factor beta $\left.(\mathrm{TGF}-\beta)^{23}\right)$; and second,

229 pathogen surface proteins for which binding proteins could have therapeutic utility

230 (Influenza A H3 hemagglutinin $(\mathrm{H} 3)^{24}$, VirB8-like protein from Rickettsia typhi (VirB8) ${ }^{25}$,

231 and the SARS-CoV-2 coronavirus spike protein) (Fig. 2a). For each target, we

232 selected one or two regions to direct binders against for maximal biological utility and for

233 potential downstream therapeutic potential. These regions span a wide range of

234 surface properties, with diverse shape and chemical characteristics (Fig. 2a and

235 Extended Data Fig. 4).

236

237

Using the above protocol, we designed 15,000-100,000 binders for each of

238 thirteen target sites on the twelve native proteins (see Methods; we chose two sites on

239 the EGF receptor). Synthetic oligonucleotides (230bp) encoding the 50-65 residue

240 designs were cloned into a yeast surface expression vector, the designs were displayed

241 on the surface of yeast, and those which bind their target enriched by several rounds of

242 fluorescence-activated cell sorting using fluorescently labelled target proteins. The

243 starting and enriched populations were deep sequenced, and the fraction of each

244 design after each sort was determined by comparing the frequency of the design in the

245 parent and child pools. From multiple sorts at different target protein concentrations, we

246 determined, as a proxy for binding $\mathrm{Kd}$ 's, the midpoint concentration $\left(\mathrm{SC}_{50}\right)$ in the binding

247 transitions for each design in the library (Extended Data Table 1 and Supplementary

248 Methods). 
To assess whether the top enriched designs for each target fold and bind as in

251 the corresponding computational design models, and to investigate the sequence

252 dependence of folding and binding, we generated high resolution footprints of the

253 binding surface by sorting site saturation mutagenesis libraries (SSMs) in which every

254 residue was substituted with each of the 20 amino acids one at a time. For the majority,

255 but not all, enriched designs, substitutions at the binding interface and in the protein

256 core were less tolerated than substitutions at non-interface surface positions (Fig. 2b,

257 Extended Data Fig. 20 \& Extended Data Fig. 5), and all the cysteines were highly

258 conserved in designs containing disulfides. The effects of each mutation on both binding

259 energy and monomer stability were estimated using Rosetta design calculations, and a

260 reasonable correlation was found between the predicted and experimentally determined

261 effect of mutations (Extended Data Fig. 6). In almost all cases, a small number of

262 substitutions were found to increase apparent binding affinity, and we generated

263 libraries combining 5-15 of these and sorted for binding under increasingly stringent

264 (lower target concentrations) conditions. Many of these affinity-enhancing substitutions

265 were mutations to tyrosine (Extended Data Fig. 7), consistent with the high relative

266 frequency of tyrosine in natural protein interfaces ${ }^{26}$. The set of affinity increasing

267 substitutions provide valuable information for improving the approach as these

268 substitutions ideally would have been identified in the computational sequence design 269 calculations (see discussion below). 
We expressed the highest affinity combinatorially-optimized binders for each target in E.coli for more detailed structural and functional characterization. All of the

273 designs were in the soluble fraction, and could be readily purified by nickel-NTA

274 chromatography. All had circular dichroism spectra consistent with the design model, 275 and most (9 out of 13 ) were stable at $95^{\circ} \mathrm{C}$ (Fig. 2d). The binding affinities for the

276 targets were assessed by biolayer interferometry, and found to range from $300 \mathrm{pM}$ to 277900 nM (Fig. 2c and Extended Data Table 2). The sequence mapping data report on

278 the residues on the design critical for binding, but only weakly on the region of the target

279 bound. We investigated this using a combination of binding competition experiments, 280 biological assays, and structural characterization of the complexes. For the nine targets 281 for which these were available, this characterization suggested binding modes 282 consistent with the design models, as described in the following paragraphs.

\section{Host protein targets involved in signaling}

The receptor tyrosine kinases TrkA, FGFR2, PDGFR, EGFR, InsulinR, IGF1R and Tie2 are key regulators of cellular processes and are involved in the development and progression of many types of cancer ${ }^{27}$. We designed binders targeting the native ligand binding sites for PDGFR, EGFR (on both domain I and domain III, the binders are 288 referred to as EGFRn_mb and EGFRc_mb respectively), InsulinR, IGF1R and Tie2, and targeting surface regions proximal to the native ligand binding sites for TrkA and FGFR2

290 (Fig. 2a and see methods for criteria). We obtained binders to all eight target sites; the 291 binding affinities of the optimized designs ranged from $\sim 1 \mathrm{nM}$ or better for TrkA and 292 FGFR2, to 860nM for IGF1R. Competition experiments with nerve growth factor (NGF), 293 Platelet Derived Growth Factor-BB (PDGF-BB), insulin, insulin growth factor-1 (IGF-1) 
294 and Angiopoietin 1 (Ang1) on yeast suggest that the binders for TrkA, PDGFR, InsulinR,

295 IGF1R and Tie2 bind to the targeted sites (Extended Data Fig. 8), consistent with the

296 computational design models. The receptor tyrosine kinase binders as monomers are

297 all expected to be antagonists, and we tested the effect on signaling through TrkA,

298 FGFR2 and EGFR of the cognate binders on cells in culture. Strong inhibition of

299 signaling by the native agonists was observed in all three cases (Fig. 3a-c, Extended

300 Data Fig. 9 and Extended Data Fig. 10).

301

302

Binding of $\mathrm{IL}-7$ to the $\mathrm{IL}-7 \alpha$ receptor subunit leads to recruitment of the $\mathrm{Yc}_{\mathrm{c}}$ receptor, forming a tripartite cytokine-receptor complex crucial to several signaling

304 cascades leading to the development and homeostasis of $\mathrm{T}$ and $\mathrm{B}$ cells $\mathrm{s}^{28}$. We obtained

305 a picomolar affinity binder for IL-7Ra targeting the IL-7 binding site, and found that it 306 blocks STAT5 signaling induced by IL-7 (Fig. 3d). We also obtained binders to CD3ठ, 307 one of the subunits of the T-cell receptor, and the signaling molecule TGF- $\beta$, which play 308 critical roles in immune cell development and activation (Fig. 2).

\section{Pathogen target proteins}

Hemagglutinin $(\mathrm{HA})$ is the main target for influenza $A$ virus vaccine and drug

311 development, and it can be genetically classified into two main subgroups, group 1 and

312 group $2^{29,30}$. The HA stem region is an attractive therapeutic epitope, as it is highly

313 conserved across all the influenza A subtypes and targeting this region can block the

314 low $\mathrm{pH}$-induced conformational rearrangements associated with membrane fusion,

315 which is essential for virus infection ${ }^{31,32}$. Neutralizing antibodies targeting the stem 316 region of group $2 \mathrm{HA}$ have been identified through screening of large B-cell libraries 
317 after vaccination or infection that neutralize both group 1 and group 2 influenza $\mathrm{A}$

318 viruses $^{33,34}$. Protein ${ }^{1,5}$, peptide p $^{35}$ and small molecule inhibitors ${ }^{36}$ have been designed to

319 bind to the stem region of group $1 \mathrm{HA}$ and neutralize the influenza $\mathrm{A}$ viruses, but none

320 recognize the group $2 \mathrm{HA}$. However, the design of small proteins or peptides that can

321 bind and neutralize both group $1 \mathrm{HA}$ and group $2 \mathrm{HA}$ has been challenging due to three

322 differences between the group $1 \mathrm{HA}$ and the group $2 \mathrm{HA}$ : first, the group $2 \mathrm{HA}$ stem

323 region is more hydrophilic, containing more polar residues, second, in group $2 \mathrm{HA}$,

324 Trp21 adopts a configuration roughly perpendicular to the surface of the targeting

325 groove, which makes the targeted groove much shallower and less hydrophobic, and

326 third, the group $2 \mathrm{HA}$ is glycosylated at Asn38 with the carbohydrate side chains

327 covering the hydrophobic groove (Extended Data Fig. 11). We used our new method to

328 design binders to H3 HA (A/Hong Kong/1/1968), the main pandemic subtype of group 2

329 influenza virus, and obtained a binder with an affinity of $320 \mathrm{nM}$ to the wild type H3 (Fig

330 2) and $28 \mathrm{nM}$ to the deglycosylated $\mathrm{H3}$ variant (N38D) (Extended Data Fig. 12a); the

331 reduction in affinity is likely due to the entropy loss of the glycan upon binding and/or the

332 steric clash with the glycan. The binder also binds to H1 HA (A/Puerto Rico/8/1934)

333 which belongs to the main pandemic subtype of group 1 influenza virus (Extended Data

334 Fig. 12b); the binding with both $\mathrm{H} 1$ and $\mathrm{H} 3$ is competed by the stem region binding

335 neutralizing antibody $\mathrm{Fl6v3}^{33}$ on the yeast surface (Extended Data Fig. 12c,d),

336 suggesting that the binder binds the hemagglutinin at the targeted site. We also

337 designed binders to the prokaryotic pathogen protein VirB8 which belongs to the type IV

338 secretion system of Rickettsia typhi, which is the causative agent of murine typhus ${ }^{25}$. 
339 We selected the surface region composed of the second and the third helices of VirB8, 340 and obtained binders with 500 pM affinity (Fig. 2).

With the outbreak of the SARS-CoV-2 coronavirus pandemic we applied our method to design miniproteins targeting the receptor binding domain of the SARS-CoV-

3442 Spike protein near the ACE2 binding site to block receptor engagement. Due to the pressing need for coronavirus therapeutics, we recently described the results of these efforts $^{37}$ ahead of those described in this manuscript; As in the case of FGFR2, IL-7Ra and VirB8, the method yielded picomolar binders, which are among the most potent compounds known to inhibit the virus in cell culture $\left(\mathrm{IC}_{50} 0.15 \mathrm{ng} / \mathrm{ml}\right)$ and subsequent animal experiments have shown that they provide potent protection against the virus in vivo $^{38}$. The modular nature of the miniprotein binders enables their rapid integration into

351 designed diagnostic biosensors for both influenza and SARS-CoV-2 binders ${ }^{39}$.

The designed binding proteins are all very small proteins ( $<65$ amino acids), and

354 many are 3-helix bundles. To evaluate their target specificity, we tested the highest 355 affinity binder to each target for binding to all other targets. There was very little cross 356 reactivity (Fig. 4a), likely due to their quite diverse surface shapes and electrostatic

357 properties (Fig. 4b). Consistent with previous observations with affibodies ${ }^{40}$, this 358 suggests that a wide variety of binding specificities can be encoded in simple helical 359 bundles; in our approach, scaffolds are customized for each target, so the specificity 360 arises both from the set of sidechains at the binding interface, and the overall shape of 361 the interface itself. 
High-resolution structural validation

High resolution structures are critical for evaluating the accuracy of computational protein designs. We succeeded in obtaining crystal structures of the

365 unbound miniprotein binders for FGFR2 and IL-7Ra, as well as the co-crystal structures

366 of the miniprotein binders of H3, TrkA, FGFR2 and IL-7Ra in complex with their targets

367 (Extended Data Table 3). The H3 binder binds to the shallow groove of the stem region of $\mathrm{HK} 68 / \mathrm{H} 3 \mathrm{HA}$ in the crystal structure as designed; the Ca root-mean-square deviation

369 (rmsd) over the entire miniprotein binder is $1.42 \AA$ using the HA as the alignment

370 reference (Fig. 5a and Extended Data Fig. 13). The binder makes extensive

371 hydrophobic interactions with HA and almost all of the designed interface side chain

372 configurations are recapticulated in the crystal structure (Fig. 5a). There is a clear

373 reorientation of the oligosaccharide at Asn38 compared with the unbound HK68/H3

374 structure (Fig. 5a and Extended Data Fig. 11; this has been observed in HK68/H3

375 structures bound with stem region neutralizing antibodies ${ }^{33,34}$ ), consistent with the

376 higher binding affinity for a deglycosylated variant (N38D) than for wild type H3 HA

377 (A/Hong Kong/1/1968) in BLI assays (Fig. 2 and Extended Data Fig. 12). The crystal

378 structure of the TrkA binder in complex with TrkA was very close to the design model

379 (Fig. 5b). After aligning the crystal structure and design model on TrkA, the Ca rmsd

380 over the entire miniprotein binder is $2.41 \AA$, and over the two interfacial binding helices

$3811.20 \AA$. The crystal structures of the FGFR2 binder by itself (Extended Data Fig. 14a)

382 and in complex with the third Ig-like domain of FGFR4 (Fig. 5c) match the design 383 models with near atomic accuracy, with Ca rmds of $0.58 \AA$ for the binder alone and $3841.87 \AA$ over the entire complex. The TrkA binder and the FGFR2 binder bind to the 
385 curved sheet side of the ligand binding domain of TrkA and FGFR4 with extensive

386 hydrophobic and polar interactions, and most of the key hydrophobic interactions as

387 well as the primarily polar interactions in the computational design models are largely

388 recapitulated in the crystal structures (Fig $\mathbf{5 b}, \mathbf{c}$ ). The binding interface partially overlaps

389 with the native ligand binding sites of nerve growth factor (NGF) and fibroblast growth

390 factor (FGF), however, the detailed sidechain interactions are entirely different in the

391 designed and native complexes (Extended Data Fig. 15a,b). For IL-7Ra, the crystal

392 structure of the monomer is close to that of the design, with a Ca rmsd of $0.63 \AA$

393 (Extended Data Fig. 14b) and the co-crystal structure with IL-7Ra also matches with

394 the design model closely, with a Ca rmsd of $2.2 \AA$ using IL-7Ra as the reference (Fig

395 5d). Both the de novo IL-7Ra binder and the native IL-7 use two helices to bind with IL-

396 7Ra, but the binding orientations are totally different (Extended Data Fig. 15c). Further

397 highlighting the accuracy of the protein interface design method, the cryoEM structures

398 of the SARS-CoV-2 binders LCB1 and LCB3 in complex with the virus are also nearly

399 identical to the design models, with Ca rmsd of $1.27 \AA$ and $1.9 \AA$ respectively 37 (Fig. 5e).

400 While we were not able yet to solve structures for the remainder of the designs, the high

401 resolution sequence footprinting (Fig. 2b, Extended Data Fig. 20 \& Extended Data

402 Table 4) and competition results suggest that the interfaces involve both the designed

403 residues and the intended regions on the target. The very close agreement between the

404 experimentally determined structures and the original design models suggests that the

405 substitutions required to achieve high affinity play relatively subtle roles in tuning

406 interface energetics; the overall structure of the complex, including the structure of the 
407 monomer binders and the detailed target binding mode, are determined by the

408 computational design procedure.

\section{Determinants of design success}

410 For our de novo design strategy to be successful, we must encode in the $\sim 60$

411 residue designed sequences both information on the folded monomer structures, and

412 on the target binding interfaces: designs which do not fold to the correct structure, or

413 which fold to the intended structures but do not bind to the target will fail. To assess the

414 accuracy with which the monomer structure must be designed, we carried out an

415 additional calculation and experiment for the IL-7Ra target. Large numbers of scaffolds

416 were superimposed onto 11 interface helical binding motifs identified in the first broad

417 design search, and sequence design was carried out as described above. There was a

418 strong correlation between the extent of binding and the RMSD to the binding motif

419 (Extended Data Fig. 16), suggesting that designed backbones must be quite accurate

420 to achieve binding. To assess the determinants of binding of the designed interfaces,

421 assuming that the designs fold to the intended monomer structures, we took advantage

422 of the large data set (810,000 binder designs and 240,000 single mutants) generated in

423 this study. Across all targets, there was a strong correlation between success rate and

424 the hydrophobicity of the targeted region (Extended Data Fig. 17), and designs

425 observed experimentally to bind their targets tended to have stronger predicted binding

426 energy, and larger contact molecular surfaces (Extended Data Fig. 18). As found

427 previously for design of protein stability ${ }^{10}$, iterative design-build-test cycles in which the

428 design method is updated at each iteration to incorporate feedback from the previous 
429 design round should lead to systematic improvement in the design methodology and

430 success rate.

\section{Conclusions}

Our success in designing nM affinity binders for 14 target sites demonstrates that

433 binding proteins can be designed de novo using only information on the structure of the

434 target protein, without need for prior information on binding hotspots or fragments from

435 structures of complexes with binding partners. The success also suggests that our

436 design pipeline provides a quite general solution to the de novo protein interface design

437 problem that goes far beyond previously described methods. However, there is still

438 considerable room for improvement. Only a small fraction of designs bind, and in

439 almost all cases, the best of these require a few additional substitutions to achieve high

440 affinity binding (Extended Data Table 2). Furthermore, the design of binders to highly

441 polar target sites remains a considerable challenge-the sites targeted here all contain at

442 least four hydrophobic residues. The datasets generated in this work -- both the

443 information on binders versus non binders, and the feedback on the effects of individual

444 point mutants on binding -- should help guide the development of methods for designing

445 high affinity binders directly from the computer with no need for iterative experimental

446 optimization. More generally, the de novo binder design method and the large data set

447 generated here provide a starting point for investigating the fundamental physical

448 chemistry of protein-protein interactions, and for developing and assessing

449 computational models of protein-protein interactions.

450 This work is a major step forward towards the longer range goal of direct 451 computational design of high affinity binders starting from structural information alone. 
452 We expect the binders created here, and new ones created with the method moving

453 forward, will find wide utility as signaling pathway antagonists as monomeric proteins

454 and as tunable agonists when rigidly scaffolded in multimeric formats, and in

455 diagnostics and therapeutics for pathogenic disease. Unlike antibodies, the designed

456 proteins can be expressed solubly in E. coli at high levels and are thermostable, and

457 hence could form the basis for a next generation of lower cost protein therapeutics.

458 More generally, the ability to rapidly and robustly design high affinity binders to arbitrary

459 protein targets could transform the many areas of biotechnology and medicine that rely

460 on affinity reagents.

\section{Acknowledgements}

462 This work was supported by DARPA Synergistic Discovery and Design (SD2)

463 HR0011835403 contract FA8750-17-C-0219 (L.C., B.C., S.H., D.B.), The Audacious

464 Project at the Institute for Protein Design (L.K), the Open Philanthropy Project Improving

465 Protein Design Fund (B.C., D.B.), funding from Eric and Wendy Schmidt by

466 recommendation of the Schmidt Futures program (I.G., L.M.), an Azure computing

467 resource gift for COVID-19 research provided by Microsoft (L.C., B.C.), the National

468 Institute of Allergy and Infectious Diseases (HHSN272201700059C, D.B., B.H., L.S.;

469 NIH R01 Al140245 to E.M.S.; NIH R01 Al150855 to I.A.W.), the National Institute on

470 Aging (R01AG063845, B.H., D.B.), the Defense Threat Reduction Agency (HDTRA1-

471 16-C-0029, D.B. E-M.S.), The Donald and Jo Anne Petersen Endowment for

472 Accelerating Advancements in Alzheimer's Disease Research (N.B.), a gift from Gates

473 Ventures (M.D.), The Human Frontier Science Program (A.Y.) and The Howard Hughes 
474 Medical Research Institute (K.M.J, K.C.G., D.B.). Use of SSRL at Stanford Linear

475 Accelerator Center (SLAC) National Accelerator Laboratory is supported by the US

476 Department of Energy Office of Science, Office of Basic Energy Sciences under

477 contract DE-AC02-76SF00515. The SSRL Structural Molecular Biology Program is

478 supported by the Department of Energy, Office of Biological and Environmental

479 Research and the National Institutes of Health, National Institute of General Medical

480 Sciences (including P41GM103393). A part of this work is based upon research

481 conducted at the Northeastern Collaborative Access Team beamlines, which are funded

482 by the National Institute of General Medical Sciences from the National Institutes of

483 Health (P30 GM124165). The Eiger 16M detector on the 24-ID-E beam line is funded by

484 a NIH-ORIP HEI grant (S10OD021527). STRW was supported by the CCR intramural

485 research program of $\mathrm{NCI}-\mathrm{NIH}$. GM/CA at the Advanced Photon Source at Argonne

486 National Laboratory has been funded by the National Cancer Institute (ACB-12002) and

487 the National Institute of General Medical Sciences (AGM-12006, P30GM138396). This

488 research used resources of the Advanced Photon Source, a U.S. Department of Energy

489 (DOE) Office of Science User Facility operated for the DOE Office of Science by

490 Argonne National Laboratory under Contract No. DE-AC02-06CH11357. The Eiger

491 16M detector at GM/CA-XSD was funded by NIH grant S10 OD012289. We thank the

492 staff of beamline ID23-2 (ESRF) for technical support and beamtime allocation. S.N.S.

493 acknowledges research support from Research Foundation Flanders (grants

494 G0C2214N and G0E1516N), and the Hercules Foundation (no. AUGE- 11-029). S.N.S.

495 is a principal investigator of the VIB (Belgium). 
497 We thank George Ueda for kindly providing the Ang1 protein for the TrkA competition 498 assay and Deborah H. Fuller for kindly providing the Fl6v3 antibody for the HA

499 competition assay. We thank Yong-Jun Park, Alexandra Walls, and David Veesler for

500 their collaborative research and cryoEM structure determination for minibinders

501 targeting SARS-CoV-2 Spike. We would also like to thank Kandise Van Wormer and

502 Austin Curtis Smith for their tremendous laboratory support during COVID-19.

\section{Competing interests:}

504 L. C., B.C., I.G., B.H., E-M.S., L.S. and D.B. are coinventors on a provisional patent 505 application that incorporates discoveries described in this manuscript.

506

507

508 


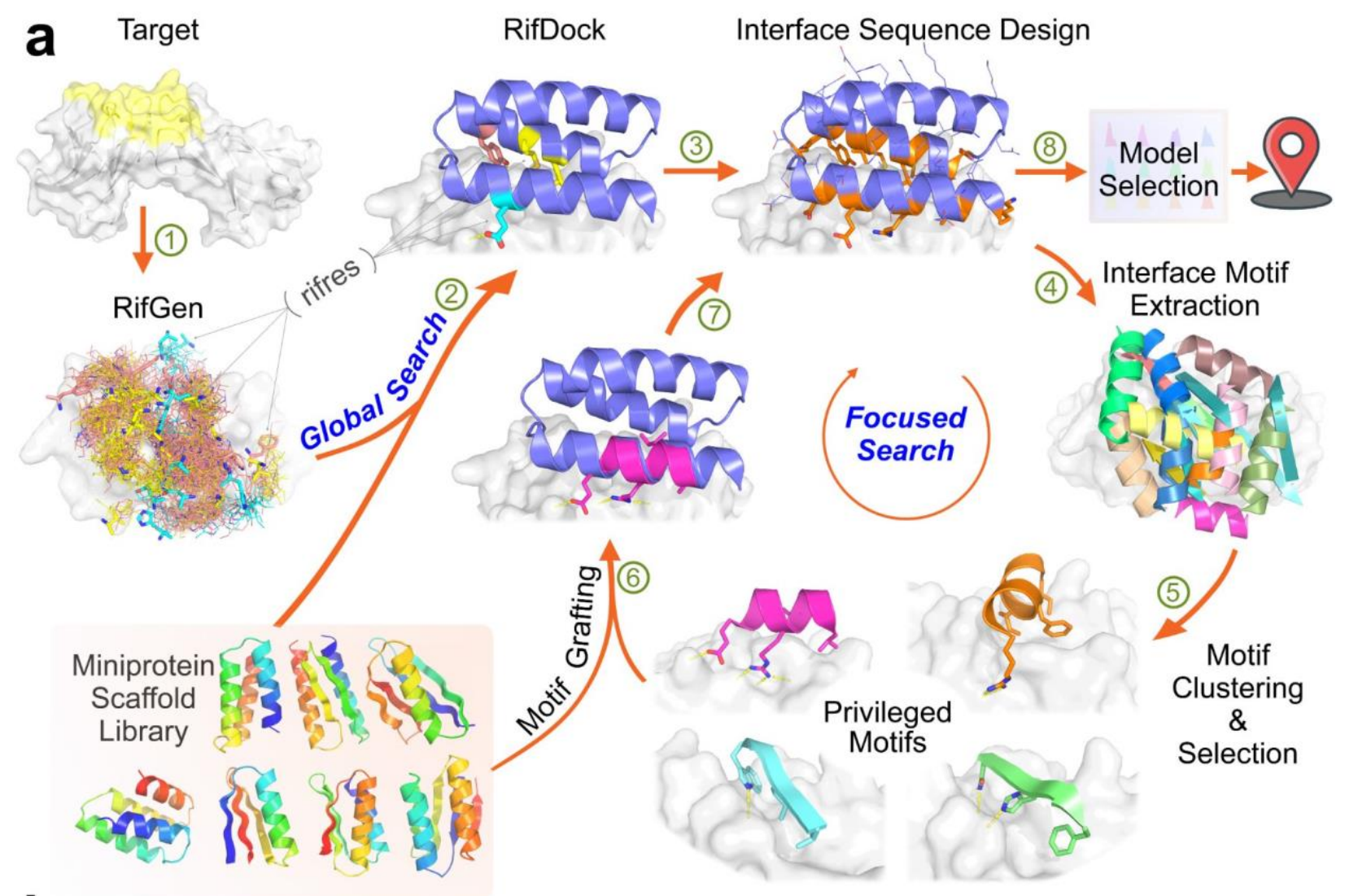

b
Contact Molecular Surface

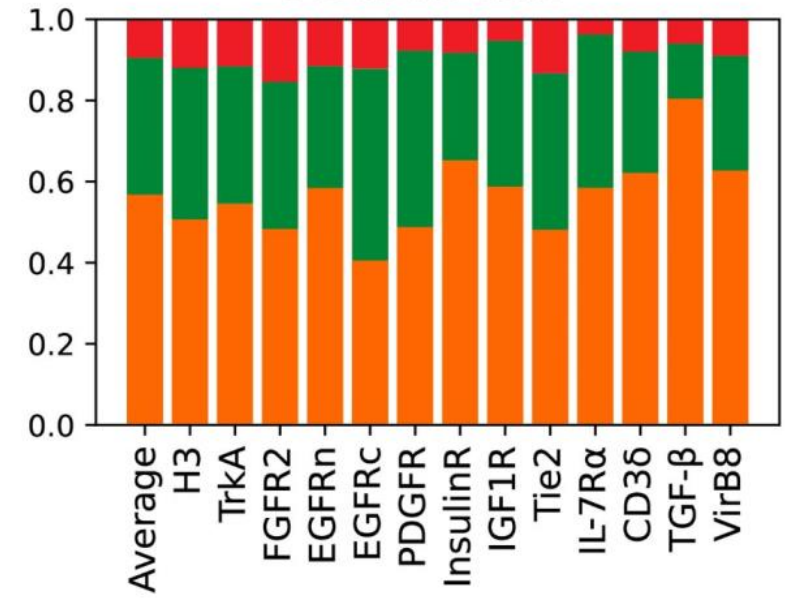

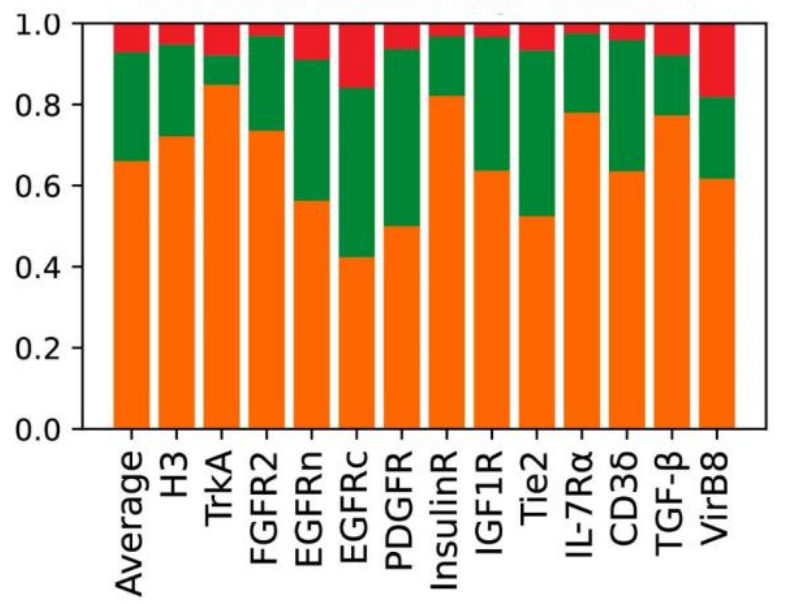

Resampling 
512 Figure 1: Overview of the de novo protein binder design pipeline. a, Schematic of 513 our two stage binder design approach. In the global search stage, billions of 514 disembodied amino acids are docked onto the selected targeting region and the 515 positioning of the scaffolds is guided by the favorable sidechain interactions. The 516 interface sequences are then designed to maximize interaction with the target. In the 517 focused search stage, the interface motifs are extracted, clustered. The privileged 518 motifs are then selected to guide another round of docking and design. Designs are 519 then selected for experimental characterization based on computational metrics. b, 520 Comparison of sampling efficiency of PatchDock, RifDock, and resampling protocols. 521 Bar graph shows the distribution over the three approaches of the top $1 \%$ of binders 522 based on Rosetta ddg and contact molecular surface after pooling equal-CPU-time 523 dock-and-design trajectories for each of the 13 target sites and averaging per-target 524 distributions (see Methods). 
bioRxiv preprint doi: https://doi.org/10.1101/2021.09.04.459002; this version posted September 5, 2021. The copyright holder for this preprint (which was not certified by peer review) is the author/funder, who has granted bioRxiv a license to display the preprint in perpetuity. It is made available under aCC-BY-NC-ND 4.0 International license.
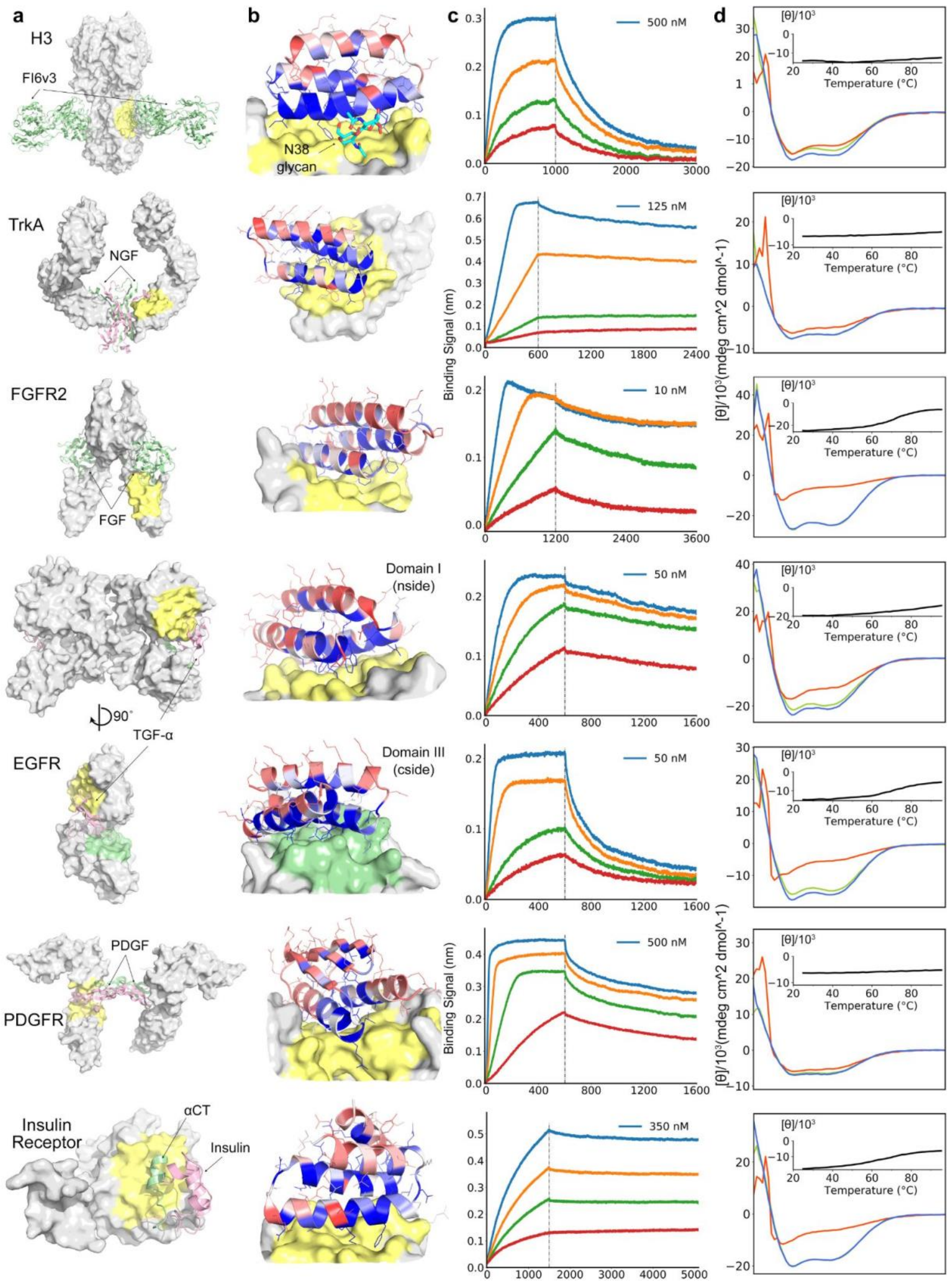

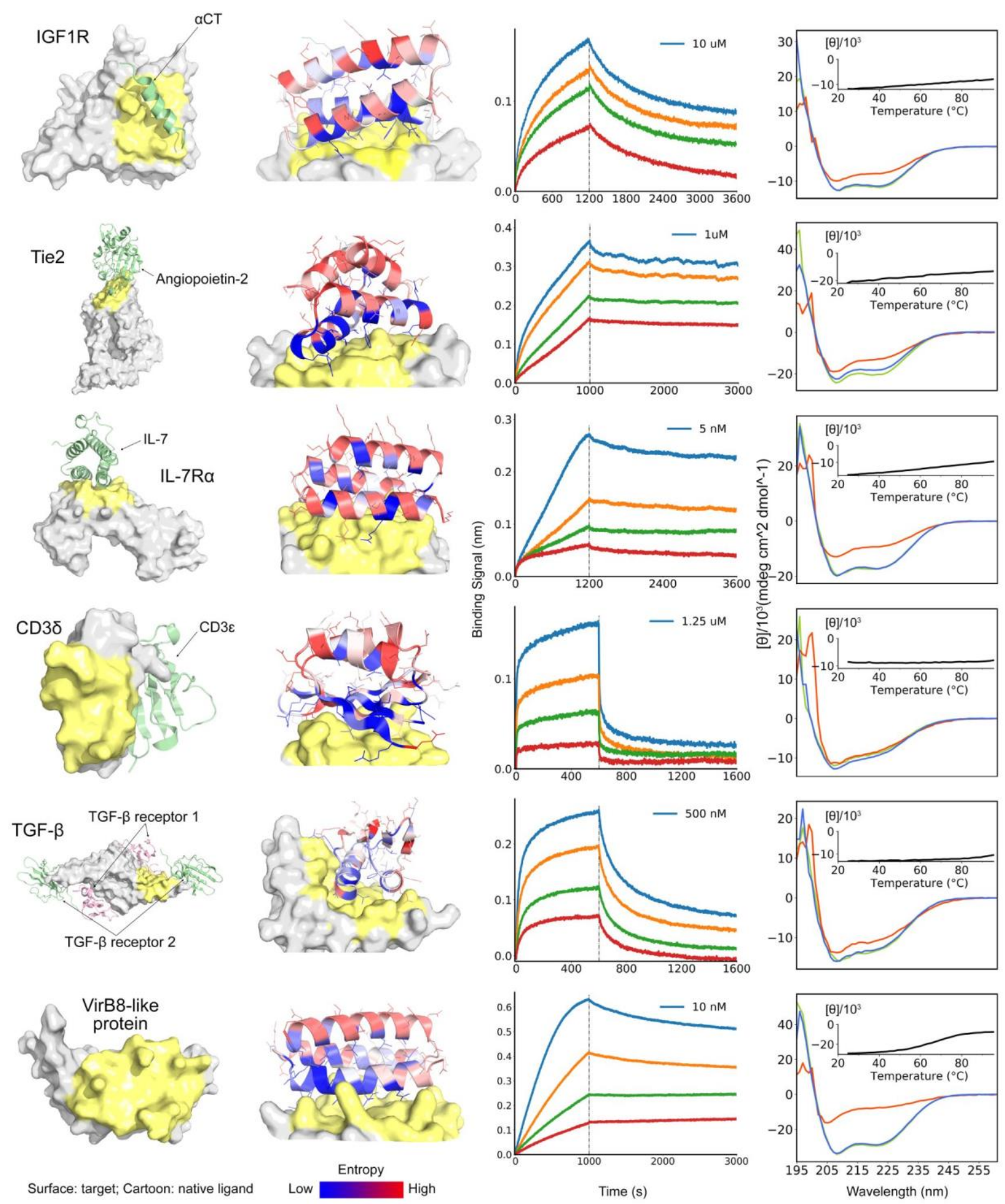

529 Figure 2: De novo design of miniprotein binders to 13 target sites. a, Naturally occurring target protein structures shown in surface representation, with known interacting partners $f$ in cartoons where available. Regions targeted for binder design or in pale yellow or green; the remainder of the target surface is in grey. See (Extended 
533 Data Figure 4) for the zoomed in views of the selected targeting regions. The PDB ID 534 codes are 3ZTJ (H3), 2IFG (TrkA), 1DJS (FGFR2), 1MOX (EGFR), 3MJG (PDGFR), 535 4OGA (InsulinR), 5U8R (IGF1R), 2GY7 (Tie2), 3DI3 (IL-7Ra), 1XIW (CD3ठ), 3KFD 536 (TGF- $\beta$ ) and 4O3V (VirB8). b, Computational models of designed complexes colored 537 by site saturation mutagensis results. Designed binding proteins (cartoons) are colored 538 by positional Shannon entropy, with blue indicating positions of low entropy (conserved) 539 and red those of high entropy (not conserved); target surface is in grey and yellow. The 540 core residues and binding interface residues are more conserved than the non-interface 541 surface positions, consistent with the computational models. Full SSM maps over all 542 positions of all the de novo designs are provided in (Supplementary file/Extended 543 Data Fig. 18). c, Biolayer interferometry characterization of binding of optimized 544 designs to the corresponding targets. Two-fold serial dilutions were tested for each 545 binder and the highest concentration is labeled. For H3, TrkA, FGFR2, EGFR, PDGFR, 546 IL-7R $\alpha, C D 3 \delta$, TGF- $\beta$ and VirB8, the biotinylated target proteins were loaded onto the 547 Streptavidin (SA) biosensors, and incubated with miniprotein binders in solution to 548 measure association and dissociation. For IGF1R and Tie2, MBP- (maltose binding 549 protein) tagged miniprotein binders were used as the analytes. For InsulinR, the 550 miniprotein binder was immobilized onto the Amine Reactive Second-Generation 551 (AR2G) Biosensors and the insulin receptor was used as the analyte. d, Circular 552 dichroism spectra at different temperatures (green: $25^{\circ} \mathrm{C}$, red: $95{ }^{\circ} \mathrm{C}$, blue: $95{ }^{\circ} \mathrm{C}$ 553 followed by $25^{\circ} \mathrm{C}$ ) and (insert) $\mathrm{CD}$ signal at 222-nm wavelength as a function of 554 temperature for the optimized designs. 
a

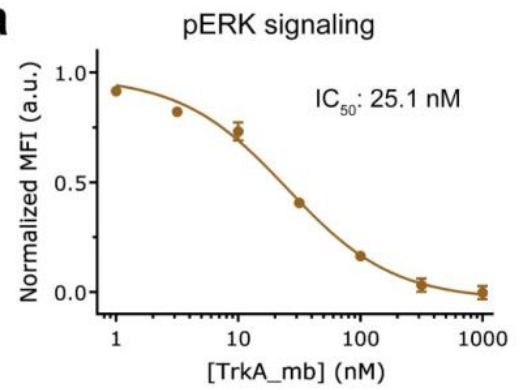

b

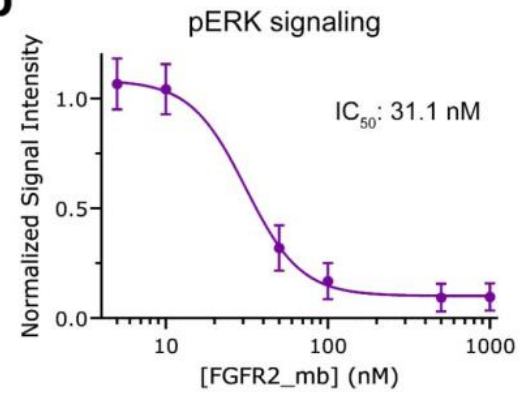

d

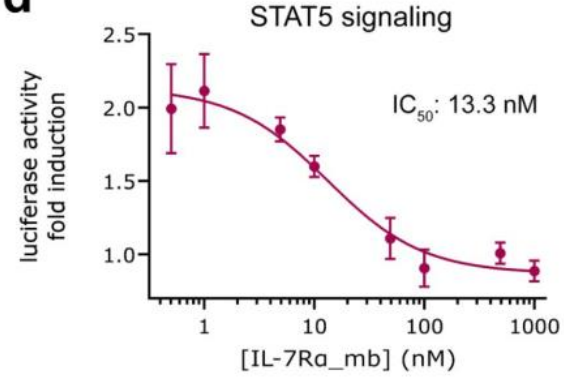

pAKT signaling

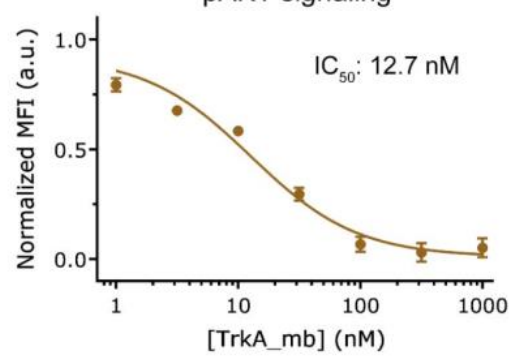

C

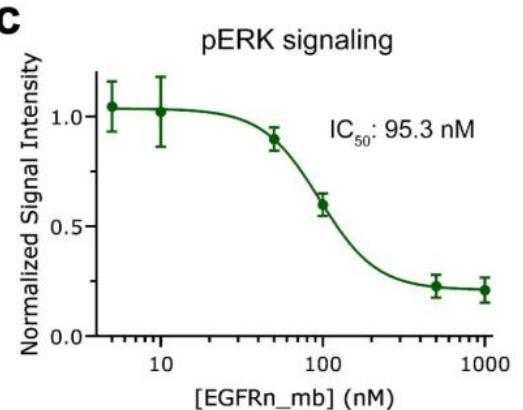

Cell proliferation

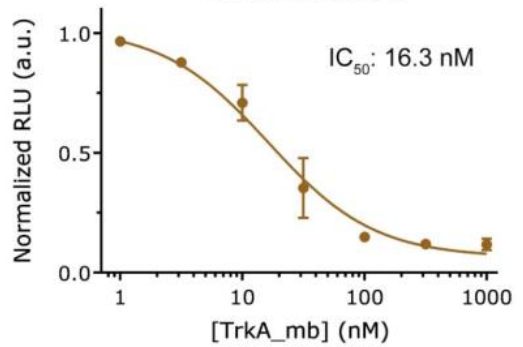

pAKT signaling

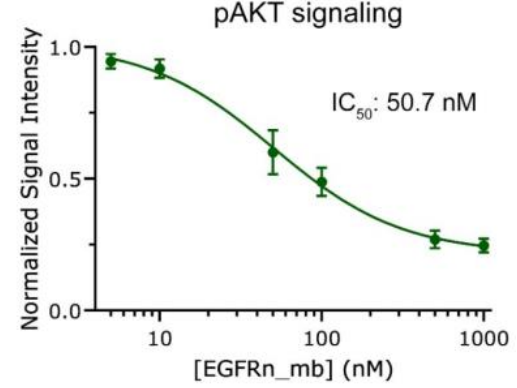

Figure 3: Inhibition of native signaling pathways by designed miniprotiens. a, Dose-dependent reduction in (left) pERK signaling, (middle) pAKT signaling and cell proliferation after $48 \mathrm{hrs}$ (right) of TF-1 cells with increase in TrkA minibinder concentration. $8.0 \mathrm{ng} / \mathrm{ml}$ human beta-NGF was used for competition. Titration curves at different concentrations of NGF and the effects of the miniprotein binders on cell viability are in Extended Data Fig. 9. b, Dose-dependent reduction pERK signaling elicited by 0.75 nM bFGF in HUVECs with increasing FGFR2 minibinder concentration. c, Dosedependent reduction in (left) pERK signaling, and (right) pAKT signaling elicited by $1 \mathrm{nM}$ EGF in HUVECs with increase in EGFR n-side minibinder concentration. See Extended Data Fig. 10 and methods for the experimental details. d, Reduction in STAT5 activity induced by 50 pM of hIL-7 in HEK293T cells in the presence of increased hIL-7Ra 

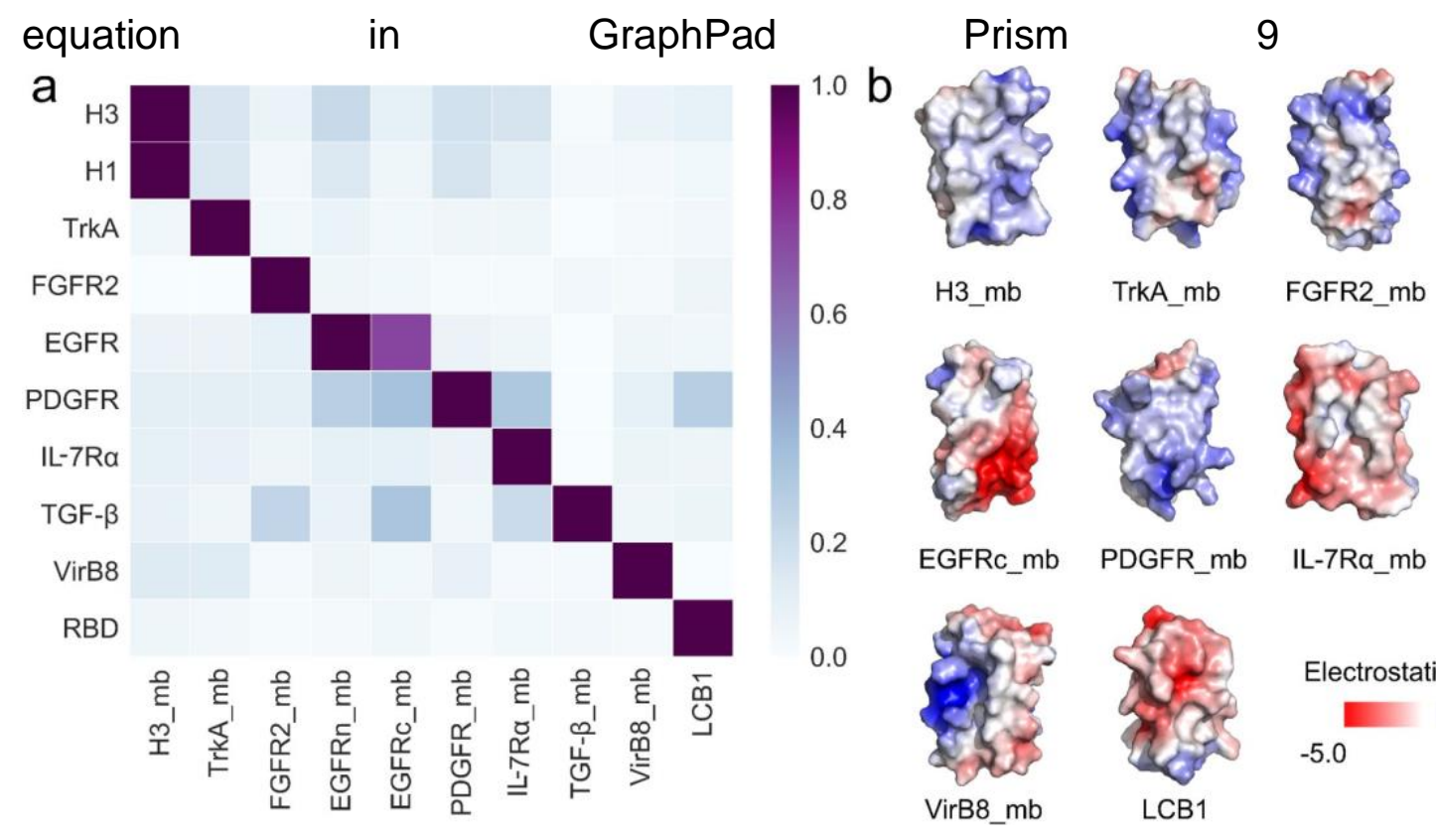

software.

H3_mb

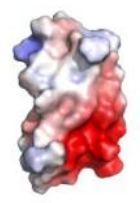

EGFRc_mb

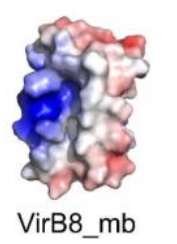

TrkA_mb

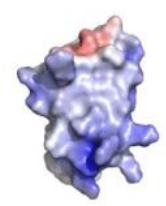

PDGFR_mb

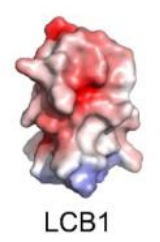

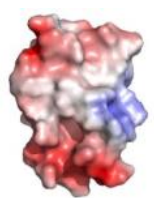

FGFR2_mb EGFRn_mb
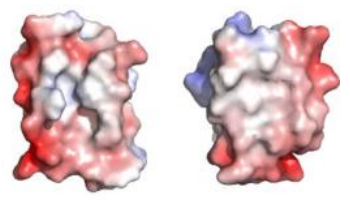

IL-7Ra_mb TGF- $\beta$ mb

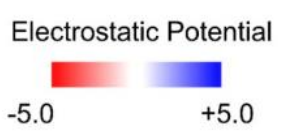

571 Figure 4: Designed binders have high target specificity. To assess the cross 572 reactivity of each miniprotein binder with each target protein, The biotinylated target 573 proteins were loaded onto biolayer interferometry SA sensors, allowed to equilibrate, 574 and baseline signal set to zero. The BLI tips were then placed into $100 \mathrm{nM}$ binder 575 solution for 300 seconds, washed with buffer, and dissociation was monitored for an 576 additional 600 seconds. Heatmap shows the maximum response signal for each binder577 target pair normalized by the maximum response signal of the cognate designed binder578 target pair. b, Surface shape and electrostatic potential (generated with the APBS 579 Electrostatics plugin in Pymol; red positive, blue, negative) of the designed binding 580 interfaces. 
a

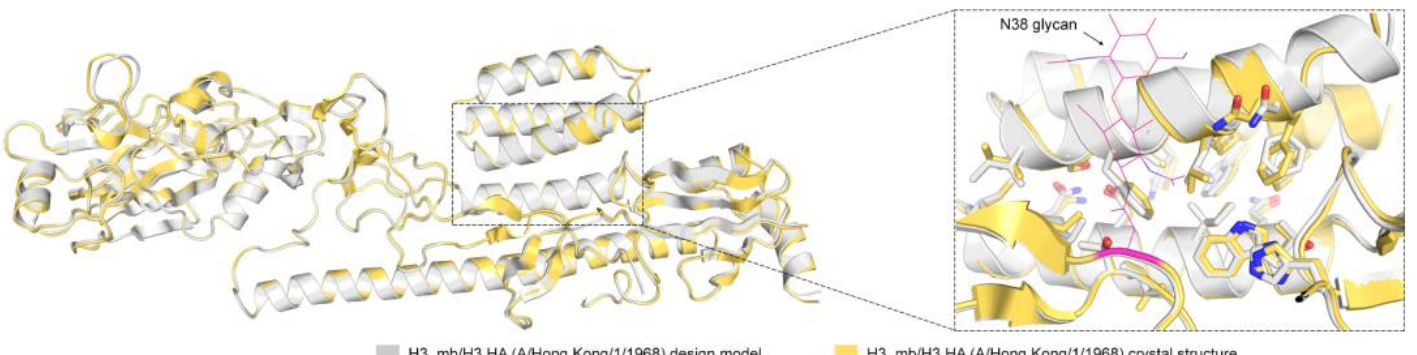

$\mathrm{b}$

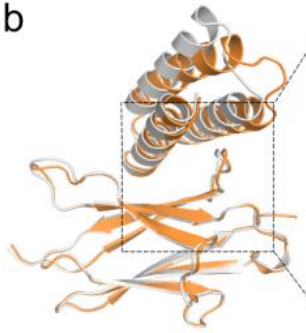

[1] TrkA_mb/TrkA design model

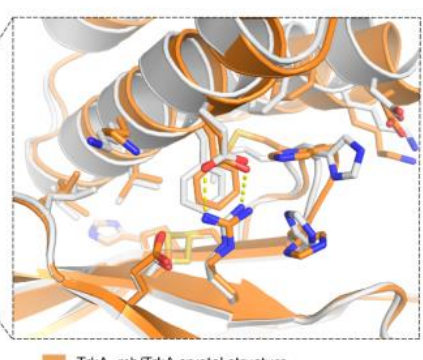

- TrkA_mb/TrkA crystal structure

C

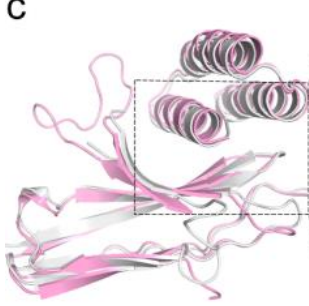

FGFR2_mb/FGFR2 design mode

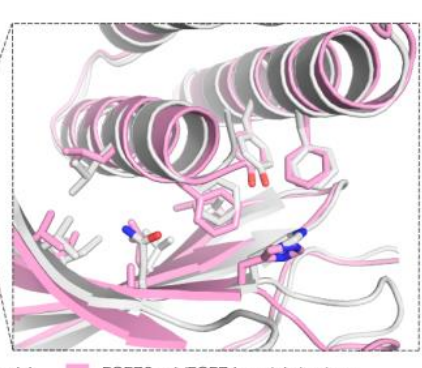

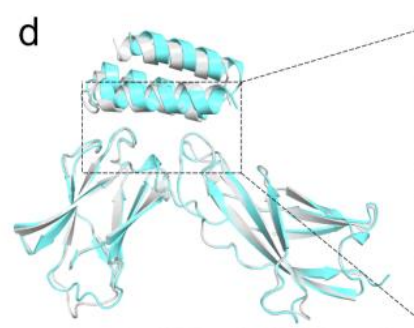

[L-7Ra_mb/L7Ra design model

e

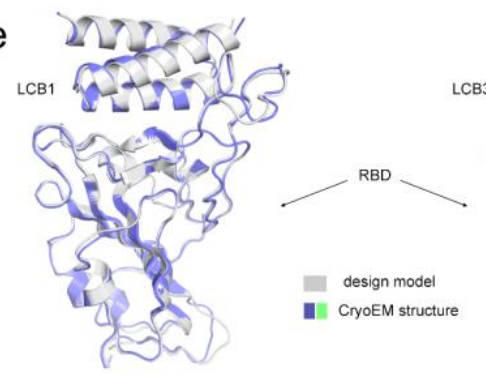

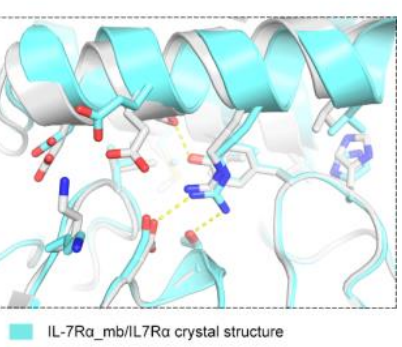

(1)

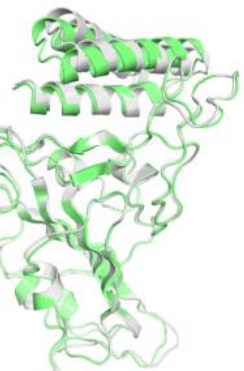

Figure 5: High-resolution structures of miniprotein binders in complex with target proteins are very close to computational design models. (a-d). (left) Superimposition of computational design model (silver) on experimentally determined crystal structure. (right) Zoom-in view of designed interface, with interacting side chains as sticks. a. H3, b. TrkA, c. FGFR2, d. IL-7Ra. e, Superimposition of the computational design model and refined cryo-EM structures of LCB1 (left) and LCB3 (right) bound to receptor binding domain of SARS-CoV-2 spike protein (design models are in gray and cryoEM structures are in pale blue and green). 


\section{Reference}

5951 Chevalier, A. et al. Massively parallel de novo protein design for targeted

$596 \quad$ therapeutics. Nature 550, 74-79, doi:10.1038/nature23912 (2017).

5972 Strauch, E. M. et al. Computational design of trimeric influenza-neutralizing

598

599

600 proteins targeting the hemagglutinin receptor binding site. Nature biotechnology 35, 667-671, doi:10.1038/nbt.3907 (2017).

601

602 Silva, D. A. et al. De novo design of potent and selective mimics of IL-2 and IL-15.

603 Nature 565, 186-191, doi:10.1038/s41586-018-0830-7 (2019).

$4 \quad$ Baran, D. et al. Principles for computational design of binding antibodies.

604

605 Proceedings of the National Academy of Sciences of the United States of

606

607

608 America 114, 10900-10905, doi:10.1073/pnas.1707171114 (2017).

5 Fleishman, S. J. et al. Computational design of proteins targeting the conserved stem region of influenza hemagglutinin. Science 332, 816-821, doi:10.1126/science.1202617 (2011).

609

6 Dou, J. et al. De novo design of a fluorescence-activating beta-barrel. Nature 561, 485-491, doi:10.1038/s41586-018-0509-0 (2018).

611 Koga, N. et al. Principles for designing ideal protein structures. Nature 491, 222227, doi:10.1038/nature11600 (2012).

8 Linsky, T. et al. Sampling of Structure and Sequence Space of Small Protein Folds. bioRxiv (2021).

9 Maguire, J. B. et al. Perturbing the energy landscape for improved packing during computational protein design. Proteins 89, 436-449, doi:10.1002/prot.26030 (2021). design, synthesis, and testing. Science 357, 168-175, doi:10.1126/science.aan0693 (2017).

12 Brunette, T. J. et al. Modular repeat protein sculpting using rigid helical junctions. Schneidman-Duhovny, D., Inbar, Y., Nussinov, R. \& Wolfson, H. J. PatchDock and SymmDock: servers for rigid and symmetric docking. Nucleic acids research 33, W363-367, doi:10.1093/nar/gki481 (2005). Proceedings of the National Academy of Sciences of the United States of America 117, 8870-8875, doi:10.1073/pnas.1908768117 (2020).

13 Coventry, B. \& Baker, D. Protein sequence optimization with a pairwise decomposable penalty for buried unsatisfied hydrogen bonds. PLOS computational biology 17, e1008061, doi:10.1371/journal.pcbi.1008061 (2021).

14 Wiesmann, C., Ultsch, M. H., Bass, S. H. \& de Vos, A. M. Crystal structure of nerve growth factor in complex with the ligand-binding domain of the TrkA receptor. Nature 401, 184-188, doi:10.1038/43705 (1999). structures of two FGF-FGFR complexes reveal the determinants of ligandreceptor specificity. Cell 101, 413-424, doi:10.1016/s0092-8674(00)80851-x (2000). receptor extracellular domain bound to transforming growth factor alpha. Cell 110, 763-773, doi:10.1016/s0092-8674(02)00940-6 (2002). 
63917 Shim, A. H. et al. Structures of a platelet-derived growth factor/propeptide complex and a platelet-derived growth factor/receptor complex. Proceedings of the National Academy of Sciences of the United States of America 107, 1130711312, doi:10.1073/pnas.1000806107 (2010).

18 Croll, T. I. et al. Higher-Resolution Structure of the Human Insulin Receptor Ectodomain: Multi-Modal Inclusion of the Insert Domain. Structure 24, 469-476, doi:10.1016/j.str.2015.12.014 (2016). $\mathrm{Xu}, \mathrm{Y}$. et al. How ligand binds to the type 1 insulin-like growth factor receptor. Nature communications 9, 821, doi:10.1038/s41467-018-03219-7 (2018).

20 Barton, W. A. et al. Crystal structures of the Tie2 receptor ectodomain and the angiopoietin-2-Tie2 complex. Nature structural \& molecular biology 13, 524-532, doi:10.1038/nsmb1101 (2006).

21 McElroy, C. A., Dohm, J. A. \& Walsh, S. T. Structural and biophysical studies of the human IL-7/IL-7Ralpha complex. Structure 17, 54-65,

22 Arnett, K. L., Harrison, S. C. \& Wiley, D. C. Crystal structure of a human CD3epsilon/delta dimer in complex with a UCHT1 single-chain antibody fragment. Proceedings of the National Academy of Sciences of the United States of America 101, 16268-16273, doi:10.1073/pnas.0407359101 (2004).

660

661

662 isoform-specific ligand recognition and receptor recruitment in the superfamily. The Journal of biological chemistry 285, 14806-14814, doi:10.1074/jbc.M109.079921 (2010).

24 Ekiert, D. C. et al. Cross-neutralization of influenza A viruses mediated by a single antibody loop. Nature 489, 526-532, doi:10.1038/nature11414 (2012).

25 Gillespie, J. J. et al. Structural Insight into How Bacteria Prevent Interference between Multiple Divergent Type IV Secretion Systems. mBio 6, e01867-01815, doi:10.1128/mBio.01867-15 (2015).

26 Birtalan, S. et al. The intrinsic contributions of tyrosine, serine, glycine and arginine to the affinity and specificity of antibodies. Journal of molecular biology 377, 1518-1528, doi:10.1016/j.jmb.2008.01.093 (2008).

27 Lemmon, M. A. \& Schlessinger, J. Cell signaling by receptor tyrosine kinases. Cell 141, 1117-1134, doi:10.1016/j.cell.2010.06.011 (2010).

28 Markovic, I. \& Savvides, S. N. Modulation of Signaling Mediated by TSLP and IL7 in Inflammation, Autoimmune Diseases, and Cancer. Frontiers in immunology 11, 1557, doi:10.3389/fimmu.2020.01557 (2020).

29 Webster, R. G., Bean, W. J., Gorman, O. T., Chambers, T. M. \& Kawaoka, Y. Evolution and ecology of influenza A viruses. Microbiological reviews 56, 152179, doi:10.1128/mr.56.1.152-179.1992 (1992).

30 Nobusawa, E. et al. Comparison of complete amino acid sequences and receptor-binding properties among 13 serotypes of hemagglutinins of influenza $A$ viruses. Virology 182, 475-485, doi:10.1016/0042-6822(91)90588-3 (1991).

682

31 Bullough, P. A., Hughson, F. M., Skehel, J. J. \& Wiley, D. C. Structure of 683 influenza haemagglutinin at the $\mathrm{pH}$ of membrane fusion. Nature 371, 37-43, doi:10.1038/371037a0 (1994). 
$68432 \quad$ Ekiert, D. C. et al. Antibody recognition of a highly conserved influenza virus epitope. Science 324, 246-251, doi:10.1126/science.1171491 (2009).

33 Corti, D. et al. A neutralizing antibody selected from plasma cells that binds to group 1 and group 2 influenza A hemagglutinins. Science 333, 850-856, doi:10.1126/science.1205669 (2011).

34 Joyce, M. G. et al. Vaccine-Induced Antibodies that Neutralize Group 1 and Group 2 Influenza A Viruses. Cell 166, 609-623, doi:10.1016/j.cell.2016.06.043 (2016).

693

35 Kadam, R. U. et al. Potent peptidic fusion inhibitors of influenza virus. Science 358, 496-502, doi:10.1126/science.aan0516 (2017).

36 van Dongen, M. J. P. et al. A small-molecule fusion inhibitor of influenza virus is orally active in mice. Science 363, doi:10.1126/science.aar6221 (2019).

37 Cao, L. et al. De novo design of picomolar SARS-CoV-2 miniprotein inhibitors. Science 370, 426-431, doi:10.1126/science.abd9909 (2020).

38 Case, J. B. et al. Ultrapotent miniproteins targeting the receptor-binding domain protect against SARS-CoV-2 infection and disease in mice. bioRxiv (2021).

701

702 Quijano-Rubio, A. et al. De novo design of modular and tunable protein biosensors. Nature 591, 482-487, doi:10.1038/s41586-021-03258-z (2021).

40 Frejd, F. Y. \& Kim, K. T. Affibody molecules as engineered protein drugs. Experimental \& molecular medicine 49, e306, doi:10.1038/emm.2017.35 (2017). 